\title{
Context Notification in Mobile Environment to Find the Right Person in Time
}

\author{
Cora Burger \\ University of Stuttgart, Institute of Parallel and Distributed High-Performance Systems \\ (IPVR), \\ Breitwiesenstr. 20-22, D-70565 Stuttgart, Germany \\ E-Mail: caburger@informatik.uni-stuttgart.de
}

\begin{abstract}
People searching for somebody else who is suited for a certain task should not only know about current location and context of this person but also about further characteristics like her capabilities. We propose a solution, that provides added value to existing services for context notification. It can be used in arbitrary networks consisting of mobile devices and stationary computers and is flexible enough for a broad spectrum of application scenarios. Two kinds of filters guarantee the appropriate level of privacy and prevent from information overflow. The prototypical realization is based on IrDA (Infrared Data Association) and a mobile agent infrastructure.
\end{abstract}

\section{Introduction}

Everybody knows the situation of looking around for consultation in a store. Having found a clerk somewhere between shelves, one will recognize that she is already busy with other customers. This problem of unsuccessful trials to find a person suited for a certain task is a very frequent one and wide spread (think e. g., of nurses and medicines in a hospital or of colleagues at office). Besides personal annoyance, it results in lost time and depending on the task in question can have serious consequences.

Mobile devices, phones and sensors bear the potential to overcome this problem and the more general one of handling arbitrary interrelationships between actions of different people most efficiently (see coordination theory in (5)). We start with taking a closer look at the above described scenarios and work out a list of five requirements that must be taken into account by any solution.

Actually, different problems can be involved in the one of finding the right person for a given task: 1. Who is suited at all? 2. Who is suited in the current situation? 3. Where is the suited person?

An answer to the first of these questions can exist, if it is known already who is suited at all. But this knowledge is not sufficient. As long as a clerk is talking to other customers she is not suited to answer questions. The same holds for some colleague who is an expert in a certain area but is currently staying overseas at some conference or has a strict deadline ahead and therefore is not in the right mood for any discussion.

It can be concluded, that a whole set of information about persons and their current environment is needed (requirement 1) and matched against characteristics of the task 
to solve the above described problem (requirement 2). Making public this information evolves the next question, the one of privacy issues. People do not want to be observed all the time and not at all by everybody else (requirement 3 ).

Another point concerns the amount of context information and frequency of notifications at the receiver's side. In the scenario of a store, this information will be queried for and delivered just once. But it can vary in degree of detail. In offices, even the frequency of being disturbed by information about colleagues plays an important role. Thus in either case, it must be possible to restrict information to a useful minimum (requirement 4).

Last but not least, context information can arise in a number of different sources: desktop or mobile devices and sensors. Furthermore, people should have information about others at hand when it is needed. Either of these cases requires integration of mobile devices. But connection to such devices is insecure and subject to frequent interruptions. Nevertheless, people should not miss essential information (requirement 5). Furthermore, information has to be protected against interception and misuse (requirement 3 again).

Current approaches to location and context awareness (e. g., (4), (7), (8), fail in the scenario of looking for a clerk, because they require a direct identification of the person in question. Furthermore, only privacy issues have been taken into account, but restriction of information overflow was neglected.

\section{Model of Enhanced Notification Service}

To properly fulfill requirement 1 , the following types of context information are essential

- Identifier, e. g. name,

- role (including capabilities and knowledge),

- location,

- $\quad$ activity and its priority.

With the exception of the identifier, these parameters vary permanently and more or less frequently. Persons can be referenced either directly by identifier or indirectly by some other characteristics. For instance, one is looking for any clerk in a store who is able to inform about a certain product. A more specific question would include further parameters, e. g., a suited clerk staying nearby without talking to anybody else and without being busy elsewhere. For any person referenced somehow, current location is of interest. Furthermore, kind of activity and degree she is currently involved in it are indicators for deciding whether this person can be disrupted or should be left in peace.

Requirement 2 is satisfied by designing an enhanced notification service with proper service elements. Context information is delivered to this service by information producers via the following service element

push (own_context, producer_filtering_conditions)

By specifying filtering conditions, the producer can determine exactly who is allowed to see which part of her context information. Thus, privacy issues are supported (requirement 3). 
For information consumers, two different sets of service elements are offered to get context information about directly or indirectly referenced producers:

pull (reference_to_person, current_context, optional:

consumer_filtering_conditions)

subscribe (reference_to_person, context_change_event, consumer_filtering_conditions) resp. modify, unsubscribe

where pull is used for singular queries (e. g. for clerk in store) and subscribe cares for constantly notifying a consumer about context parameters of the producer (e. g. at office). In all cases, the parameter reference_to_person either contains the identifier of the person (direct reference) or some characteristics and optionally selection or preference criteria (indirect reference). Furthermore, the consumer can specify filtering conditions to avoid information overflow (requirement 4).

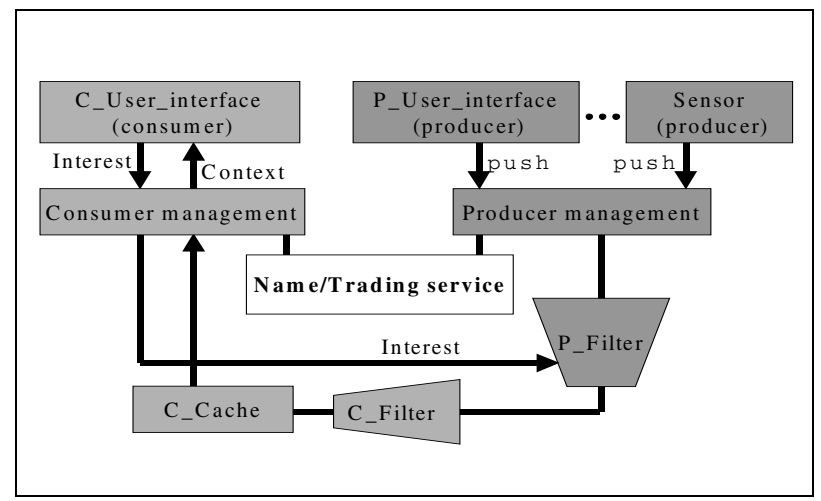

Fig. 1. General notification service

A notification service with functionality as described above must contain components as shown in figure 1. At her user interface, a person in the role of a consumer describes her interest in the form of pull or subscribe with direct or indirect reference to another person. Further treatment of this interest is handled by the corresponding consumer management. In case of direct reference by person's identifier, the relevant producer manager can be found via name service. For indirectly referenced persons by parameters like role, location or activity, a trading service (cf. (6) has to be used for mapping these characteristics to the most suited producer manager by taking into account selection and preference criteria (requirement 2). For instance, consumer management invokes

import (role=clerk_responsible_for_computers,

location=nearest, activity=free)

to find someone for consultation on computers in a store.

Depending on the form of interest (pull or subscribe), either an isolated query or a demand for registering is propagated to the P_Filter of the referenced person where it is accepted or rejected depending on the producer's privacy needs. In case of acceptance, filtering conditions contained in the interest result in setting up a corresponding C_Filter. This covers a consumer's interest in a subset of possible context information. 
A user in the role of producer addresses the management responsible for her to define criteria for filtering and customize a P_Filter. The producer manager of a person must offer its service to a trader at initiation time, e. g. by

export (identifier=Sheila, role=clerk_responsible_for_computers,

location=callback_location, activity=callback_activity)

For an incoming import request the trader asks for current values of location and activity by means of callback methods as specified in export. Whereas for queries and registries of consumers filtering criteria are applied, traders are informed about all values of context information without any restrictions. Privacy issues are preserved nonetheless, because the trading service does deliver mere references of suited producer management to requesting consumer management components.

Current values of context information are either created by the user, some application or some sensor. Via producer manager they are propagated to the corresponding P_Filter that distributes them to all C_Filters of authorized consumers. Depending on its user's interest, a C_Filter either deletes the information or transmits it to the C_Cache. This component is responsible for reliable information transfer to consumer management taking into account the possibility of connection interrupt. That means, information is stored as long as the consumer management is not connected (requirement 5).

It should be noted that because of insecure environment all parties must authenticate and encrypt data before transmitting them (requirement 3 ).

\section{Implementation}

There are several alternatives to implement the notification service as described in the last section. In the following, we present a realization based on an infrastructure for mobile agents. A mobile agent consists of mobile code. While migrating through networks, it is acting almost autonomously. Infrastructures for mobile agents enable life cycle, mobility and communication of agents. Furthermore, they guarantee restricted access to resources and cope with heterogeneity of systems involved.

For instance, the Mole agent system (1) provides an infrastructure for mobile agents written in the programming language JAVA. Its logical structure is defined by locations and two different kinds of agents: system agents and mobile agents. System agents are bound to a certain location and care for secure access to stationary resources. Mobile agents can move between locations to perform their tasks. Communication between agents takes place by means of remote procedure call or messages. Agents can create and start mobile agents.

In (2) a first approach to context notification was presented that was based on the Mole platform. It was restricted to stationary desktop computers and collaborative settings with direct references to other people. In the meantime, this system was extended to be used on handheld devices with Windows CE and connections via IrDA (Infrared Data Association). Furthermore, we introduce the possibility of guest users and indirect references via role to cope with scenarios like the one in a store as well. 


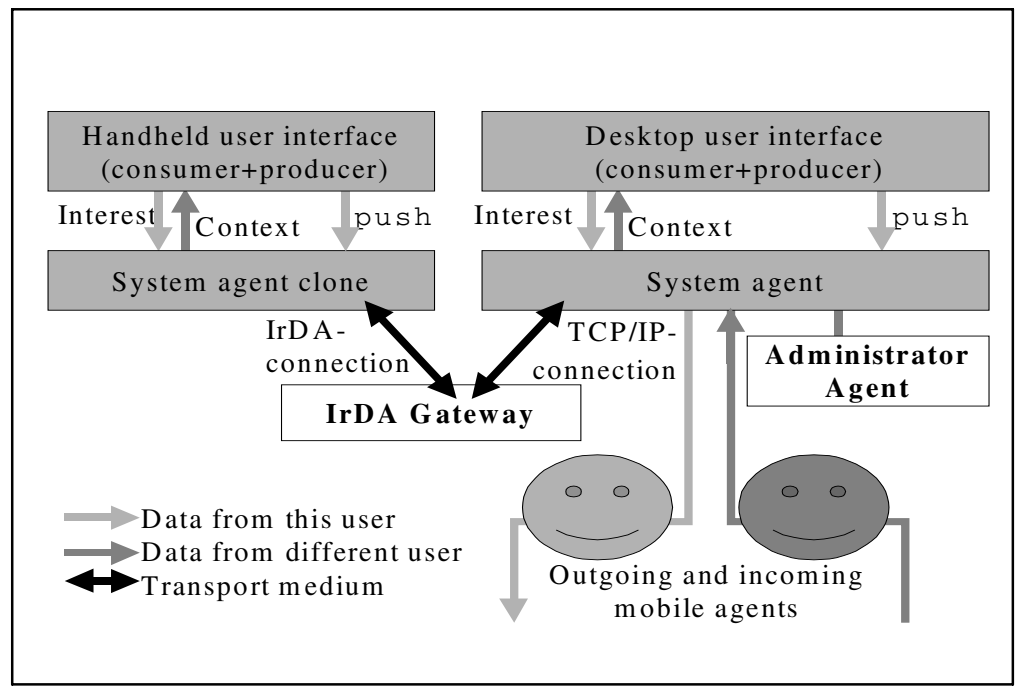

Fig. 2. Notification service in mobile environment

The new architecture is shown in figure 2. It consists of the following components (please refer to (3) for further detail):

- For each user: two different versions of user interface, one for handheld devices and one for desktop devices. They are responsible for presenting context information about other people (consumer part) and for letting users input current context information about themselves (producer part).

- For each user or for the ensemble of guests: system agent in the fixed part of the network, e. g. on desktop device. After registry at the administration agent, it manages interests of its corresponding user interfaces by sending out suited mobile agents to system agents of other users (consumer part), accepts or rejects mobile agents of other users and propagates context information from its user interface to accepted mobile agents according to its filtering criteria (producer part).

- For each pair of users, where one is interested in context information about the other one: mobile agent being created by the system agent in consumer role and transmitting interest and filtering criteria to the system agent in producer role. If the mobile agent is accepted, it filters context information delivered by the foreign system agent and propagates it to its system agent.

- For each user with handheld device: system agent clone to enable configuration of filters and exchange information between user interface on handheld device and system agent of this user via some IrDA gateway.

- On stationary devices being equipped with IrDA-interface: IrDA gateway cares for transmitting data between a system agent clone that is contacting the gateway and the corresponding system agent (or guest system agent).

- Administrator agent, current access point to name service. It is enriched to offer trader functionality also. 


\section{Conclusion and Outlook}

We have presented a system, that contributes to finding people that must not be known personally but can be referenced by characteristics like their role (requirement 1 and 2). It offers extensive configuration of filtering and security mechanisms to satisfy needs of consumers and producers of context information especially in a mobile environment (requirement 3 to 5).

One of the shortcomings of the current implementation is manual insertion of context information that is rather cumbersome. To avoid this overhead, the user interface of producers can be restricted to assigning priorities to activities and specification of filtering criteria whereas the rest is replaced by suited location services and further services to detect current activity.

As a whole, our notification service for context information increases the probability of finding the right person at right time. It can be extended in several directions. For instance, search for objects can be included and general coordination decisions of people can be facilitated.

\section{References}

(1) Baumann, J. et al.: Mole - Concepts of a Mobile Agent System. WWW Journal, Special issue on Applications and Techniques of Web Agents, 1998. http://www.informatik.unistuttgart.de/ipvr/vs/projekte/ mole.html

(2) Burger, C.: Team awareness with mobile agents in mobile environments. Proceedings of the 7th International Conference on Computer Communications and Networks (IC3N'98), IEEE Computer Society, Los Alamitos, October 1998, pp. 45-49

(3) Kramer, A.: Reachability notification of colleagues by means of mobile devices (in german). Diploma thesis No. 1777, University of Stuttgart, November 1999

(4) Leonhardt, U.: Supporting Location-Awareness in Open Distributed Systems. PhD thesis, University of London, 1998

(5) Malone, T.W. et al.: What is Coordination Theory and How Can It Help Design Cooperative Work Systems? CSCW '90 Conference on Computer Supported Cooperative Work, ACM Press, October 1990, pp. 357-370

(6) ODP Trading Function: Specification. ISO/IEC IS 13235:1 | Draft Rec . X.950:1 (1997). http://ftp.dstc.edu.au/AU/research_news/odp/trader/trtute/trtute.html

(7) Prinz, W.: NESSIE: An Awareness Environment for Cooperative Settings. Proc. $6^{\text {th }}$ European Conference on Computer Supported Cooperative Work ECSCW'99, Kluwer Academic Publishers, Dordrecht, 1999, pp. 391-410

(8) Roussopoulos, M. et al.: Person-level Routing in the Mobile People Architecture. Proceedings of the USENIX Symposium on Internet Technologies and Systems, October 1999 